\title{
Experimental constraints on the coupling of the Higgs boson to electrons
}

\author{
Wolfgang Altmannshofer, ${ }^{a}$ Joachim Brod ${ }^{b}$ and Martin Schmaltz ${ }^{a}$ \\ ${ }^{a}$ Perimeter Institute for Theoretical Physics, \\ 31 Caroline Street North, Waterloo, ON, N2L 2Y5, Canada \\ ${ }^{b}$ PRISMA Cluster of Excellence and Mainz Institute for Theoretical Physics, \\ Johannes-Gutenberg-Universität, \\ Staudingerweg 7, 55099 Mainz, Germany \\ ${ }^{c}$ Physics Department, Boston University, \\ 590 Commonwealth Ave., Boston, MA 02215, U.S.A. \\ E-mail: waltmannshofer@perimeterinstitute.ca, \\ joachim.brod@uni-mainz.de, schmaltz@bu.edu
}

ABSTRACT: In the standard model (SM), the coupling of the Higgs boson to electrons is real and very small, proportional to the electron mass. New physics could significantly modify both real and imaginary parts of this coupling. We discuss experiments which are sensitive to the Higgs-electron coupling and derive the current bounds on new physics contributing to this coupling. The strongest constraint follows from the ACME bound on the electron electric dipole moment (EDM). We calculate the full analytic two-loop result for the electron EDM and show that it bounds the imaginary part of the Higgs-electron coupling to be less than $1.7 \times 10^{-2}$ times the SM electron Yukawa coupling. Deviations of the real part are much less constrained. We discuss bounds from Higgs decays, resonant Higgs production at electron colliders, Higgs mediated $B \rightarrow e^{+} e^{-}$decays, and the anomalous magnetic moment of the electron. Currently, the strongest constraint comes from $h \rightarrow e^{+} e^{-}$ at the LHC, bounding the coupling to be less than $\sim 600$ times the SM Yukawa coupling. Important improvements can be expected from future EDM measurements as well as from resonant Higgs production at a next-generation high-luminosity electron-positron collider.

KeYwords: Higgs Physics, Beyond Standard Model, CP violation

ArXiv EPrint: 1503.04830 


\section{Contents}

1 Introduction 1

2 The Higgs-electron coupling beyond the SM 2

3 Constraints from direct searches 5

3.1 Higgs decays at the LHC and beyond 5

$\begin{array}{lll}3.2 & \text { Higgs production at electron-positron colliders } & 6\end{array}$

4 Precision constraints $\quad 8$

4.1 Electric dipole moment of the electron $\quad 9$

$\begin{array}{ll}4.2 & \text { Anomalous magnetic dipole moment of the electron } \\ \end{array}$

$\begin{array}{lll}4.3 & \text { Rare } B \text { decays } & 10\end{array}$

5 Discussion and conclusions $\quad 11$

$\begin{array}{ll}\text { A Two-loop contributions to dipole moments } & 13\end{array}$

B Enhanced Higgs production through a loop hole? 15

\section{Introduction}

The announcement of the five-sigma discovery of the Higgs boson at the LHC on July 4th 2012 officially launched a new program of precision tests of the standard model (SM) in Higgs physics. By precisely measuring the production and decay rates of the Higgs boson we aim to test if the Higgs' couplings agree with SM predictions, and — if they don't agree - we hope to obtain hints about physics beyond the SM.

So far, the focus has been on the largest couplings of the Higgs such as the couplings to $W$ and $Z$ gauge bosons as well as the top, bottom and $\tau$ Yukawa couplings $[1,2]{ }^{1}$ In this article, we concentrate instead on the coupling of the Higgs to electrons which is of course predicted to be one of the smallest couplings of the Higgs in the SM. We ask what we know about the electron Yukawa coupling from a purely experimental point of view. It might be reasonable to expect that new physics in the Higgs sector couples more strongly to top quarks than to electrons; however, measuring the Higgs coupling to electrons is interesting precisely because the SM prediction for the Yukawa coupling is so small. A higher-dimensional operator from new physics can easily compete with the SM Yukawa coupling or can even dominate.

\footnotetext{
${ }^{1}$ Recently, methods for measuring first and second generation quark Yukawa couplings were proposed as well $[3-5]$.
} 
In section 2 we briefly discuss how higher-dimensional operators can modify the coupling of the Higgs to electrons. In section 3 we analyze the sensitivity to a modified Higgs-electron coupling coming from searches for Higgs decays into $e^{+} e^{-}$at the LHC and at future hadron colliders, as well as from Higgs production at electron-positron colliders. Indirect constraints on a modified Higgs-electron coupling from the electric dipole moment $(\mathrm{EDM})$ and the anomalous magnetic dipole moment (MDM) of the electron, as well as from rare $B$-meson decays into $e^{+} e^{-}$final states are discussed in section 4 . We conclude in section 5 with a summary of current and future constraints. In appendix A we provide analytic expressions for the complete set of relevant two-loop contributions to the electron EDM and MDM that are induced by a modified Higgs-electron coupling. In appendix B we show that in the Standard Model the Higgs electron coupling is necessarily suppressed by the electron mass to all loop orders.

\section{The Higgs-electron coupling beyond the SM}

Within the SM, both the electron mass $m_{e}$ and the Higgs-electron coupling $g_{e e h}$ are completely determined by the Yukawa coupling $y_{e}$ of the first generation leptons to the Higgs doublet $\varphi$,

$$
\mathcal{L}_{\mathrm{SM}} \supset y_{e}^{\mathrm{SM}} \bar{\ell}_{L} \varphi e_{R}+\text { h.c. } .
$$

After electroweak symmetry breaking, we can parametrize $\varphi=\left(G^{+},\left(v+h+i G^{0}\right) / \sqrt{2}\right)^{T}$ and thus obtain the electron mass term and the coupling of the physical Higgs boson $h$ to left and right handed electrons:

$$
\mathcal{L} \supset m_{e} \bar{e}_{L} e_{R}+\frac{g_{e e h}}{\sqrt{2}} \bar{e}_{L} e_{R} h+\text { h.c. . }
$$

Given the known electron mass $m_{e} \simeq 0.511 \mathrm{MeV}$ and the Higgs vacuum expectation value (vev) $v=\left(\sqrt{2} G_{F}\right)^{-1 / 2} \simeq 246 \mathrm{GeV}$, one can predict the Higgs-electron coupling in the SM

$$
g_{e e h}^{\mathrm{SM}}=y_{e}^{\mathrm{SM}}=\sqrt{2} m_{e} / v \simeq 2.9 \times 10^{-6} .
$$

We see that the Higgs-electron coupling is of the order of $10^{-6}$ and real. At the quantum level, the magnitude of the coupling receives well-known perturbative corrections starting at order $y_{e} \alpha$, where $\alpha$ is the fine-structure constant, and requires a proper definition of the quantities involved. We will ignore all such complications because the experimental uncertainties will turn out to be much larger than the change in the coupling due to running. In appendix B we give a general proof based on chiral symmetry showing that all quantum corrections to the Higgs-electron coupling in the SM are proportional to the electron mass and are therefore small.

How can the coupling of the Higgs to electrons differ from the value predicted in eq. (2.3)? Assuming that the field content of the SM provides an adequate description of physics at the weak scale, any new physics contributions can be parametrized by higherdimensional operators respecting the SM gauge symmetries. Thus, to modify the Higgselectron coupling, we must introduce a higher-dimensional operator coupling the Higgs to electrons that changes the relationship between the electron mass and the Yukawa coupling. 

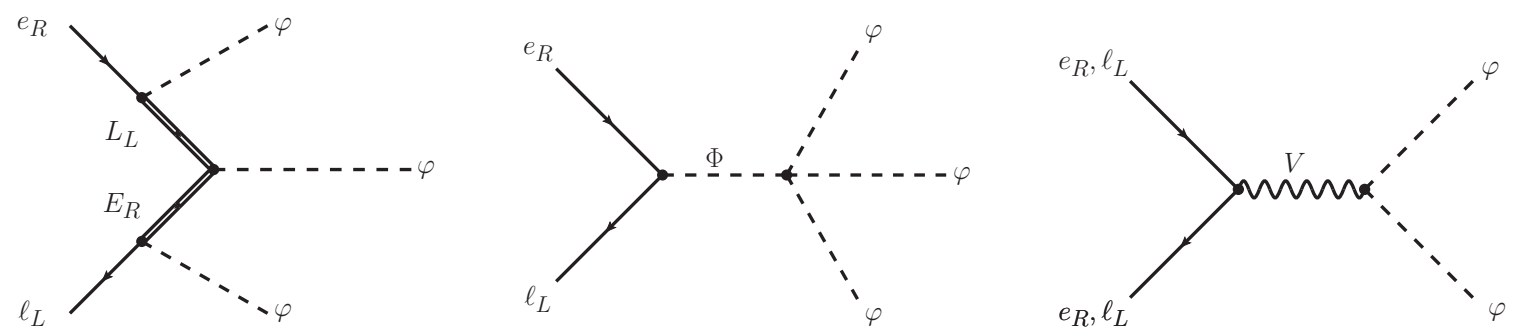

Figure 1. Possible origins of the dimension-six operator in eq. (2.4). Left: mixing of the electrons with heavy vector-like leptons. Middle: mixing of the SM Higgs doublet with a heavy scalar doublet that couples to electrons. Right: exchange of a heavy vector.

The lowest-dimension operators which do this are of dimension six and have zero, one, or two derivatives

$$
\begin{aligned}
\mathcal{L}_{\operatorname{dim} 6} \supset & \frac{c_{0}}{M^{2}} \varphi^{\dagger} \varphi \bar{\ell}_{L} \varphi e_{R}+\text { h.c. } \\
& +\frac{c_{1 L}}{M^{2}} \bar{\ell}_{L} \gamma^{\mu} \ell_{L} \partial_{\mu}\left(\varphi^{\dagger} \varphi\right)+\frac{c_{1 L}^{\prime}}{M^{2}} \bar{\ell}_{L} \gamma^{\mu} \ell_{L}\left(\varphi^{\dagger} \stackrel{\leftrightarrow}{D} \mu\right)+\left(\ell_{L} \leftrightarrow e_{R}\right) \\
& +\frac{c_{2}}{M^{2}} \bar{\ell}_{L} e_{R} D^{2} \varphi+\ldots
\end{aligned}
$$

where $M$ is a new-physics scale, $c_{0}$ and $c_{2}$ are complex couplings, and the $c_{1}$ couplings are real. Such operators could arise from mixing of the leptons with heavy vector-like fermions, from mixing of the Higgs with a heavy scalar doublet, or from the exchange of new vector bosons (see figure 1). Generically, we would expect that the couplings $c_{i}$ are $3 \times 3$ matrices in lepton flavor space such that the operators in eq. (2.4) not only modify the Higgs-electron coupling but also alter the other Higgs-lepton couplings, thereby also inducing lepton-flavor violating Higgs couplings. Possible relations among the new physics effects in these couplings are, however, model dependent and their discussion is beyond the scope of this work.

We now argue that the only dimension-six operator which can significantly modify the coupling of on-shell electrons to the Higgs is the one proportional to $c_{0}$. To start, note that the operators in the second line preserve chiral symmetry and might therefore be expected to have a larger coefficient than those in the first and third line which do break chiral symmetry. Yet, the operators proportional to $c_{1 L}^{\prime}$ and $c_{1 R}^{\prime}$ do not contribute to the Higgselectron coupling. The operators proportional to $c_{1 L}$ and $c_{1 R}$ do modify the real part of the Higgs-electron coupling; however, after integrating by part and applying the equations of motion one sees that these contributions are suppressed by a factor $m_{e} / M$ in addition to $v / M$ and are therefore too small to be interesting. In addition, there are several potentially interesting two-derivative operators, but only the one shown in the third line of eq. (2.4) gives contributions to the Higgs-electron coupling which are not suppressed by powers of the electron mass. In Higgs production or decay, the Higgs boson is on shell and the derivatives can simply be replaced by $M_{h}^{2}$, thus allowing the effects of this operator to be absorbed by a shift of $c_{0}$. For low-energy experiments the derivatives get replaced by small momenta and the effects of the $c_{2}$ operator are negligible. We will therefore concentrate on 
the $c_{0}$ operator as a plausible source of observable deviations in the Higgs-electron coupling from now on.

Expanding the Higgs doublet about its vev, the $c_{0}$ operator in eq. (2.4) leads to corrections to the electron mass and the Higgs-electron coupling of order $v^{2} / M^{2}$ :

$$
\begin{aligned}
m_{e} & =\frac{v}{\sqrt{2}}\left(y_{e}+\frac{c_{0}}{2} \frac{v^{2}}{M^{2}}\right), \\
g_{e e h} & =y_{e}+\frac{3 c_{0}}{2} \frac{v^{2}}{M^{2}}=\frac{\sqrt{2} m_{e}}{v}+c_{0} \frac{v^{2}}{M^{2}} .
\end{aligned}
$$

Note the factor of 3 in the ratio of the new physics contributions to $g_{e e h}$ and $m_{e}$ relative to the SM contributions. In the presence of both the SM Yukawa coupling and the dimensionsix operator, the Higgs-electron coupling and the electron mass become independent parameters. ${ }^{2}$ As $m_{e} \ll v$, the new physics correction to the Higgs-electron coupling can be sizable, even for very large new physics scales $M \gg v$. However, one should keep in mind that $g_{e e h} \gg g_{e e h}^{\mathrm{SM}}$ is only possible if there is a significant cancellation between the contributions to the electron mass coming from the Yukawa coupling and the higher-dimensional operator, cf. eq. (2.5).

Note that given the smallness of the electron mass, operators of dimension greater than 6 may also play a role in determining the Higgs-electron coupling and the electron mass. For instance, in the models by Giudice and Lebedev $[6] g_{e e h}$ is dominated by contributions from dimension-ten operators, and could be (naturally) a factor of $\mathcal{O}(10)$ larger than the SM prediction.

Finally we point out that $g_{e e h}$ can in general be complex; a non-vanishing imaginary part of $g_{e e h}$ would be a clear sign of new physics. For a sizable phase to arise there have to be at least two different operators contributing to $g_{e e h}$, with coefficients of similar magnitude and different phases (for instance, the dimension-four and -six contributions in eq. (2.5) with $y_{e} \sim c_{0} v^{2} / M^{2}$ ). The electron mass term can always be made real by an appropriate choice of the phase of the electron fields. The Higgs-electron interaction then has, in general, a complex phase relative to the mass term.

In order not to commit ourselves to a specific scenario, we find it convenient to parametrize the modified Higgs-electron coupling more generally as

$$
g_{e e h}=\kappa_{e} \frac{\sqrt{2} m_{e}}{v},
$$

where $\kappa_{e}$ is a complex parameter describing the relative deviation from the SM prediction $\kappa_{e}^{\mathrm{SM}}=1$. In the case that only dimension-six operators are relevant, we can use the relation $\kappa_{e}=1+c_{0} v^{3} /\left(\sqrt{2} m_{e} M^{2}\right)$ together with the assumption that $c_{0}$ is a coefficient of order unity to translate a bound on $\kappa_{e}$ into a lower bound on the NP scale $M$.

Throughout this article we set all couplings of the Higgs boson to particles other than the electron to their SM values.

\footnotetext{
${ }^{2}$ On the other hand, if one can neglect contributions of operators with mass dimension higher than six, the effective couplings of electrons to more than one Higgs boson are fixed in terms of $g_{e e h}$ and $m_{e}$.
} 


\section{Constraints from direct searches}

The coupling of the Higgs to electrons leads to the decay of the Higgs into electrons. Moreover, it allows resonant production of Higgs bosons in electron-positron collisions in the $s$-channel. In this section we will discuss the sensitivity to a modified Higgs-electron coupling of searches for $h \rightarrow e^{+} e^{-}$decays at hadron colliders and of $s$-channel Higgs production at electron-positron colliders.

\subsection{Higgs decays at the LHC and beyond}

The recent search for SM Higgs decays in the $\mu^{+} \mu^{-}$and $e^{+} e^{-}$channels by CMS [7] allows to set a bound on the Higgs-electron coupling. Modifying the Higgs-electron coupling will change both the $h \rightarrow e^{+} e^{-}$partial width and the total Higgs decay width. Accordingly, we find for the modified branching ratio

$$
\operatorname{Br}\left(h \rightarrow e^{+} e^{-}\right)=\frac{\left|\kappa_{e}\right|^{2} \operatorname{Br}\left(h \rightarrow e^{+} e^{-}\right)_{\mathrm{SM}}}{1+\left(\left|\kappa_{e}\right|^{2}-1\right) \operatorname{Br}\left(h \rightarrow e^{+} e^{-}\right)_{\mathrm{SM}}},
$$

where we neglected terms that are further suppressed by $m_{e}^{2} / M_{h}^{2}$. For a Higgs mass of $M_{h}=125.7 \mathrm{GeV}$ [8], the SM prediction for the branching ratio reads [9]

$$
\operatorname{Br}\left(h \rightarrow e^{+} e^{-}\right)_{\mathrm{SM}} \simeq 5.1 \times 10^{-9} .
$$

Assuming the SM Higgs production cross section, CMS finds an upper bound on the branching ratio of $[7]$

$$
\operatorname{Br}\left(h \rightarrow e^{+} e^{-}\right)<0.0019 \text { @ 95\% C.L. . }
$$

This results in the constraint

$$
\left|\kappa_{e}\right|<611 .
$$

Setting the new physics coupling of the dimension-six operator in (2.4) to $c_{0}=1$ we can translate the constraint on $\kappa_{e}$ into a constraint on the new physics scale $M$. We find $M>5.8 \mathrm{TeV}$. We expect that the bound on $\kappa_{e}$ from $h \rightarrow e^{+} e^{-}$can be improved in the future at the LHC. The gluon fusion Higgs production cross section increases by approximately by a factor 2.5 going from $8 \mathrm{TeV}$ to $14 \mathrm{TeV}[9,10]$. Assuming that the sensitivity to the $h \rightarrow e^{+} e^{-}$decay scales with the square root of the number of Higgs events, we expect sensitivities to $\left|\kappa_{e}\right| \sim 260$ with $300 / \mathrm{fb}$ and $\left|\kappa_{e}\right| \sim 150$ with $3 /$ ab. At a $100 \mathrm{TeV}$ proton-proton collider the Higgs production cross section increases by another factor of $\sim 15$ [11]. An integrated luminosity of 3/ab might allow to improve the sensitivity down to $\left|\kappa_{e}\right| \sim 75$.

We close this subsection by considering the Higgs two-body decay to positronium and a photon. This decay is the electron analogue of the Higgs decays to vector meson and photon which were recently studied [3-5] to measure the Higgs couplings to light quarks. The idea behind this method is that the vector meson plus gamma final state can result from two different amplitudes which interfere. One of the amplitudes involves the Higgs coupling to the light quarks in the vector meson, the other amplitude generates the vector meson via mixing with a virtual photon. Naively, these two amplitudes have different chiral symmetry 

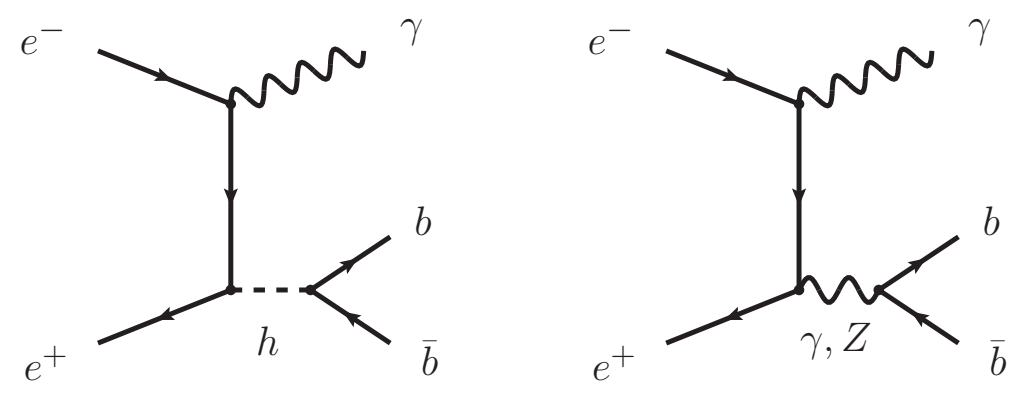

Figure 2. Resonant Higgs boson production at LEP II via radiative return to the Higgs pole (left diagram). The Higgs is assumed to decay into a $b \bar{b}$ final state. The main background is given by off-shell photons or $Z$ bosons decaying into a $b \bar{b}$ pair (right diagram).

properties and cannot interfere. However, chiral symmetry is broken dynamically by the QCD condensate, allowing the interference term to be proportional to only one power of the small quark Yukawa coupling. The case of Higgs decay to positronium is quite analogous except that here the only source of chiral symmetry breaking is the electron mass so that the interference term is necessarily proportional to the electron mass (times powers of alpha) in addition to the Higgs-electron coupling. Thus this final state is less sensitive to the Higgs-electron coupling than the $h \rightarrow e^{+} e^{-}$decay considered above.

\subsection{Higgs production at electron-positron colliders}

The electron Yukawa coupling allows for resonant production of Higgs bosons in $e^{+} e^{-}$ collisions in the $s$-channel. While the cross section for this process is obviously maximized when the center of mass energy is tuned to the Higgs mass, one can also obtain sensitivity to $\kappa_{e}$ from virtual Higgs exchange or through "radiative return". Radiative return occurs when the center of mass energy of the collider exceeds the Higgs mass; in this case bremsstrahlung off an initial electron can reduce the effective center-of-mass (CM) energy to the Higgs resonance.

For instance, LEP II accumulated an integrated luminosity of the order of $500 \mathrm{pb}^{-1}$ per experiment at a few different CM energies above the Higgs pole [12] so that the radiative return process was possible. To obtain a rough estimate on the reach of the LEP II experiments we approximate the radiative return cross section simply as a $t$-channel process (that ignores some logarithmic enhancement for initial-state radiation photons) with the Higgs decaying into a $b \bar{b}$ pair (see figure 2). We use madgraph [13] to calculate the corresponding cross section $\sigma_{\text {r.r. }}$, restricting the invariant mass of the $b \bar{b}$ pair to the Higgs mass within the LEP II jet energy resolution $\sigma_{E \text {,jet }}=10 \mathrm{GeV}$ [14]. We further assume that the main background is provided by virtual photons and $Z$ bosons decaying into a $b \bar{b}$ pair in the same invariant-mass bin, with a cross section $\sigma_{\mathrm{bkg}}$.

We collect the cross sections for various $\mathrm{CM}$ energies, as well as the corresponding integrated luminosities per experiment, in table 1. Adding all data sets, we find $N_{\text {r.r. }}=$ $3 \cdot 10^{-6} \times\left|\kappa_{e}\right|^{2}$ and $N_{\mathrm{bkg}}=121$ for the total number of signal and background events, respectively. Setting $N_{\text {r.r. }} / \sqrt{N_{\mathrm{bkg}}}=1$ we see that LEP II was, in principle, sensitive to $\left|\kappa_{e}\right| \sim 2000$. We find that a similar sensitivity could be obtained with the $20 / \mathrm{pb}$ that have 


\begin{tabular}{|cccc|}
\hline$E[\mathrm{GeV}]$ & $\mathcal{L}[1 / \mathrm{pb}]$ & $10^{6} /\left|\kappa_{e}\right|^{2} \times \sigma_{\text {r.r. }}[\mathrm{fb}]$ & $\sigma_{\text {bkg }}[\mathrm{fb}]$ \\
\hline 189 & 170 & 1.40 & 56.9 \\
192 & 30 & 1.33 & 54.2 \\
196 & 80 & 1.25 & 50.8 \\
200 & 80 & 1.18 & 47.4 \\
202 & 40 & 1.14 & 45.8 \\
205 & 80 & 1.08 & 43.4 \\
207 & 140 & 1.04 & 41.9 \\
\hline
\end{tabular}

Table 1. The integrated luminosity $\mathcal{L}$ collected by each experiment at LEP II at various CM energies $E$, and the corresponding cross sections for producing a photon, plus a $b \bar{b}$ pair with an invariant mass between $115 \mathrm{GeV}$ and $135 \mathrm{GeV}$, via a virtual Higgs $\left(\sigma_{\text {r.r. }}\right)$ or an off-shell photon or $Z$ boson $\left(\sigma_{\mathrm{bkg}}\right)$.

been collected much closer to the Higgs resonance at a center of mass energy of $130 \mathrm{GeV}$. Our rough sensitivity estimates are weaker than the LHC bound derived in the previous section and for example do not take into account signal efficiencies or backgrounds from fakes. The LHC bound is expected to improve significantly after run II. Thus, a more sophisticated analysis of the LEP II data does not seem worth while.

On the other hand, resonant Higgs production would be possible at a potential future electron-positron collider running at a CM energy tuned to the Higgs mass. The cross section for the production of a massless fermion-antifermion pair via an $s$-channel Higgs is given by

$$
\sigma_{e^{+} e^{-} \rightarrow h \rightarrow f \bar{f}}(s)=\frac{1}{32 \pi} \frac{\left(y_{e}^{\mathrm{SM}}\right)^{2} y_{f}^{2}}{4} N_{c}^{f}\left|\kappa_{e}\right|^{2} \frac{s}{\left(s-M_{h}^{2}\right)^{2}+\Gamma_{h}^{2} M_{h}^{2}},
$$

where $N_{c}^{f}$ is the color factor for the final state fermions $\left(N_{c}^{f}=3\right.$ for quarks and $N_{c}^{f}=1$ for leptons). In the SM, the width of a $125.7 \mathrm{GeV}$ Higgs is $\Gamma_{h}^{\mathrm{SM}}=4.17 \mathrm{MeV}[9]$. Due to the tiny SM $h \rightarrow e^{+} e^{-}$branching fraction the change in the total width of the Higgs for $\kappa_{e} \neq 1$ is completely negligible, given currently allowed values of $\kappa_{e}$. Indeed, from the constraint in eq. (3.4) we find

$$
\Delta \Gamma_{h}=\Gamma_{h}^{\mathrm{SM}} \times\left(\left|\kappa_{e}\right|^{2}-1\right) \operatorname{Br}\left(h \rightarrow e^{+} e^{-}\right)_{\mathrm{SM}}<7.9 \mathrm{keV} .
$$

In order to calculate the resonant cross section we need to convolve the parton-level cross section $\sigma\left(e^{+} e^{-} \rightarrow h \rightarrow f \bar{f}\right)$ with the beam energy resolution. We take it as a Gaussian with variance $\Delta \equiv R \sqrt{s} / \sqrt{2}$, where $R$ is the percentage beam energy resolution [15]. Using $R=0.05 \%$ [16] and assuming an average center of mass energy exactly at the Higgs mass we find the following signal cross section for bottom quarks in the final state

$$
\sigma_{\text {sig }}\left(e^{+} e^{-} \rightarrow h \rightarrow b \bar{b}\right) \simeq\left|\kappa_{e}\right|^{2} \times 0.05 / \mathrm{fb} .
$$

For $100 / \mathrm{fb}$ of data at the Higgs resonance, this corresponds to approximately $N_{\mathrm{sig}} \simeq 5 \times\left|\kappa_{e}\right|^{2}$ signal events. 


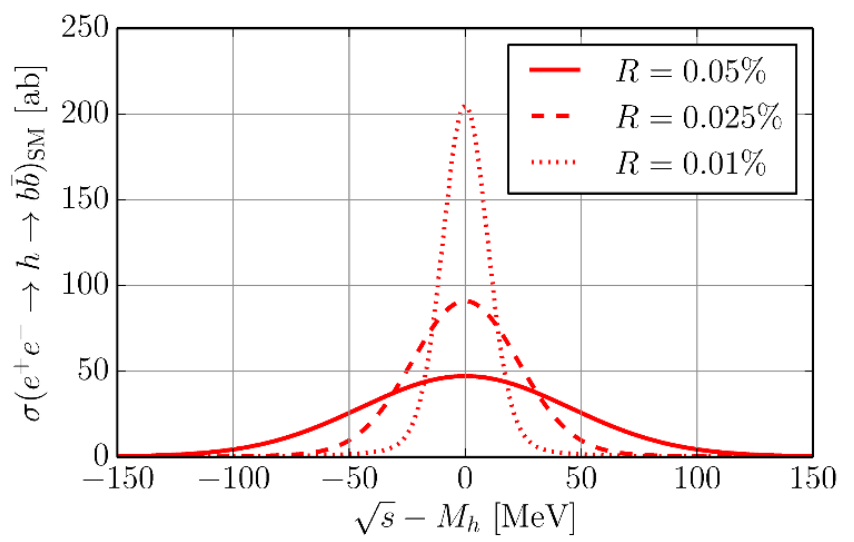

Figure 3. Dependence of the $e^{+} e^{-} \rightarrow h \rightarrow b \bar{b}$ cross section on the CM mass energy of the initial electron-positron pair. Depending on the beam energy spread $R$, the Higgs mass has to be known within a few to tens of $\mathrm{MeV}$ to fully exploit resonant production.

The main background will be $f \bar{f}$ production via an intermediate photon or $Z$ boson. The corresponding total cross section is [17]

$$
\sigma_{e^{+} e^{-} \rightarrow \gamma, Z \rightarrow f \bar{f}}(s)=\frac{4 \pi \alpha^{2}}{3 s} N_{c}^{f}\left[Q_{f}^{2}+\frac{\left(v_{e}^{2}+a_{e}^{2}\right)\left(v_{f}^{2}+a_{f}^{2}\right) s^{2}-2 v_{e} v_{f} Q_{f} s\left(s-M_{Z}^{2}\right)}{\left(s-M_{Z}^{2}\right)^{2}+\Gamma_{Z}^{2} M_{Z}^{2}}\right] .
$$

The parameters $v_{f}$ and $a_{f}$ are the vector and axial-vector couplings of the $Z$ boson to a fermion $f$. They are given by

$$
v_{f}=\frac{I_{3}^{f}-2 Q_{f} \sin ^{2} \theta_{w}}{2 \sin \theta_{w} \cos \theta_{w}}, \quad a_{f}=\frac{I_{3}^{f}}{2 \sin \theta_{w} \cos \theta_{w}},
$$

where $I_{3}^{f}$ and $Q_{f}$ denote the third isospin component and the electric charge of the fermion $f$, respectively. Assuming again 100/fb of data and $\sqrt{s}=M_{h}$ we expect roughly $N_{\text {bkg }}=10^{6} b \bar{b}$ background events. Requiring $N_{\mathrm{sig}} / \sqrt{N_{\mathrm{bkg}}}=1$ we estimate that one can reach sensitivity to $\left|\kappa_{e}\right| \lesssim 15$ for $100 / \mathrm{fb}$ and to $\left|\kappa_{e}\right| \lesssim 50$ for $1 / \mathrm{fb}$. Slightly better sensitivities could be achieved with a smaller beam energy spread.

Note that, in order to exploit the full benefit of resonant Higgs production, the Higgs mass has to be known with high precision. Figure 3 shows the $e^{+} e^{-} \rightarrow h \rightarrow b \bar{b}$ cross section as a function of the center of mass energy of the initial state electrons for three choices of the beam energy resolution $R=0.05 \%, R=0.025 \%$, and $R=0.01 \%$. The cross-section drops quickly if the center of mass energy differs from the Higgs mass by more than a few to tens of $\mathrm{MeV}$, depending on the beam energy spread.

\section{Precision constraints}

We have seen that the LHC sensitivity to the Higgs electron coupling is unlikely to reach values better than $\left|\kappa_{e}\right| \simeq 100$ whereas a future electron-positron collider running on the Higgs resonance could be sensitive to $\left|\kappa_{e}\right|$ of order 10. In addition to these direct searches, low energy precision observables can be used to indirectly probe modified Higgs couplings. Constraints from low-energy flavor observables on flavor-violating fermion-Higgs couplings 

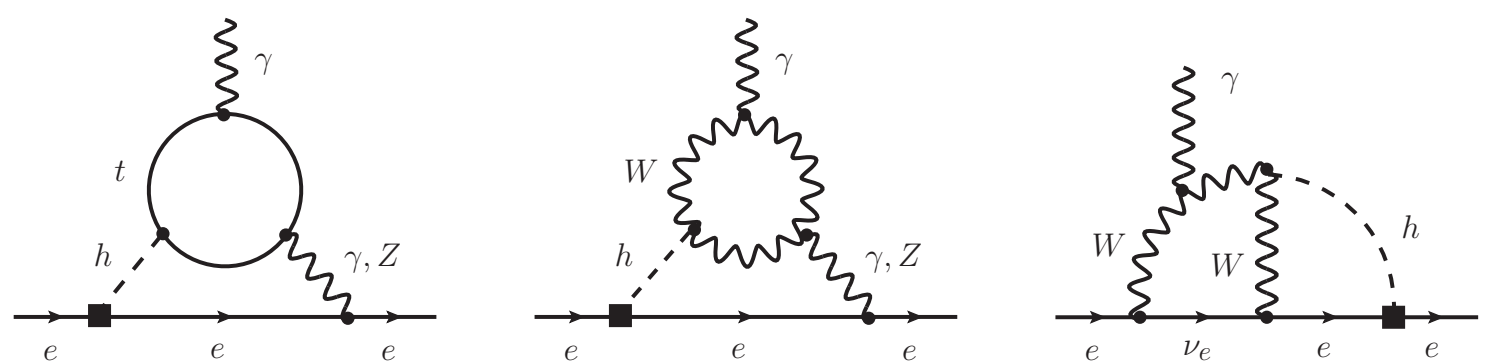

Figure 4. Sample two-loop Feynman diagrams inducing an EDM for the electron through a CPviolation Higgs coupling to the electron (here denoted by the black square).

have been derived for example in [18-20]. Constraints from EDMs on CP violating topHiggs and photon-Higgs couplings are discussed for example in [21-23].

In this section we investigate indirect constraints on a modified Higgs-electron coupling. We will see that the strongest constraints arise from the electric and magnetic dipole moments of the electron, whereas rare $B$ decays into $e^{+} e^{-}$final states do not yield competitive bounds. Note that the indirect constraints derived in this section hold barring accidental cancellations with additional contributions to the low energy observables that might arise in explicit models that give rise to the higher-dimensional operators modifying the Higgs couplings. Here we assume that all couplings other than the Higgs-electron coupling are SM-like.

\subsection{Electric dipole moment of the electron}

The imaginary part of the Higgs boson coupling to electrons in eq. (2.6) induces an EDM of the electron ${ }^{3}$

$$
\mathcal{L}_{\text {eff }}^{e}=-\frac{d_{e}}{2} \bar{\psi}_{e} \sigma_{\mu \nu} i \gamma_{5} \psi_{e} F^{\mu \nu}
$$

via two-loop electroweak diagrams ${ }^{4}$ (see figure 4 for sample Feynman diagrams). We have calculated the full set of relevant two-loop contributions that contain exactly one power of the Higgs-electrons coupling. The analytic expressions can be found in appendix A. Taking the numerical values of the input parameters $\left(\alpha, M_{W}, M_{Z}, M_{h}, m_{t}\right)$ from ref. [8] we obtain

$$
\left|\frac{d_{e}}{e}\right| \simeq 5.1 \times\left|\operatorname{Im} \kappa_{e}\right| \times 10^{-27} \mathrm{~cm} .
$$

Using the most recent bound on the electron EDM obtained by the ACME collaboration [25],

$$
\left|\frac{d_{e}}{e}\right|_{\exp }<8.7 \times 10^{-29} \mathrm{~cm} @ 90 \% \text { C.L. }
$$

we find the very stringent constraint

$$
\left|\operatorname{Im} \kappa_{e}\right|<1.7 \times 10^{-2} .
$$

\footnotetext{
${ }^{3}$ We define $\sigma_{\mu \nu}=i\left[\gamma_{\mu}, \gamma_{\nu}\right] / 2$

${ }^{4}$ One-loop contributions are suppressed by additional powers of the electron Yukawa and electron mass, and are therefore negligibly small [24].
} 
If the new physics contribution to the Higgs-electron coupling contains an $\mathrm{O}(1)$ phase, this bound translates into a very strong constraint on the new physics scale $M$. Setting $c_{0}=i$ (in the basis where the electron mass term is real) we find $M \gtrsim 1000 \mathrm{TeV}$. It is expected that the experimental sensitivity to the electron EDM can be improved by up to two orders of magnitude in the future [26]. Such sensitivities would allow to probe $\left|\operatorname{Im} \kappa_{e}\right|$ at the level of $10^{-4}$ and new physics scales as high as $10^{4} \mathrm{TeV}$.

\subsection{Anomalous magnetic dipole moment of the electron}

The real part of $\kappa_{e}$ modifies the SM contribution to the anomalous magnetic dipole moment of the electron,

$$
\mathcal{L}_{\text {eff }}^{m}=-\frac{e}{4} \frac{a_{e}}{m_{e}} \bar{\psi}_{e} \sigma_{\mu \nu} \psi_{e} F^{\mu \nu}
$$

via the same two-loop diagrams that induce also an EDM (see figure 4 and appendix A). Denoting the contributions of the two-loop diagrams with an anomalous Higgs coupling by $\Delta a_{e}$, we find

$$
\left|\Delta a_{e}\right| \simeq 2.6 \times\left(\operatorname{Re} \kappa_{e}-1\right) \times 10^{-16} .
$$

The anomaly in the gyromagnetic ratio of the electron, $a_{e} \equiv(g-2)_{e} / 2$, is conventionally used to determine the fine-structure constant $\alpha[27,28]$. However, as pointed out in ref. [29], the recent precise independent measurements of the fine-structure constant in atomic physics experiments can be used to obtain a SM prediction for $a_{e}$ with an uncertainty that is only a factor of few larger than the experimental measurement. Therefore, the anomalous magnetic moment of the electron can be used as a probe of new physics.

We employ the value $\alpha^{-1}=137.035999037(91)$ from the most recent determination of the fine-structure constant using a measurement of the ratio between the Planck constant and the mass of the ${ }^{87} \mathrm{Rb}$ atom [30]. Using the corresponding uncertainty induced on $a_{e}$ around the SM value, we obtain the allowed range for the new physics contribution to $a_{e}$

$$
\left|\Delta a_{e}\right|<8.1 \times 10^{-13}
$$

This translates into the allowed range for $\kappa_{e}$,

$$
\left|\operatorname{Re} \kappa_{e}\right|<3.1 \times 10^{3} \text {. }
$$

This is a factor of five above the direct bound derived from the CMS search for $h \rightarrow e^{+} e^{-}$. Note, however, that this bound scales linearly with Re $\kappa_{e}$, in contrast to the quadratic dependence of the collider constraints. The bound from the anomalous magnetic moment can be improved in the near future by an order of magnitude [29], making it competitive to the expected sensitivities from $h \rightarrow e^{+} e^{-}$at run II of the LHC.

\subsection{Rare $B$ decays}

In the Standard Model, the rare decays $B_{q} \rightarrow \ell^{+} \ell^{-}$are mediated by $Z$-penguin and box diagrams and require a helicity flip of the final state leptons due to the pseudo-scalar nature of the $B_{q}$ meson. Therefore, the branching ratios are proportional to the lepton mass 
squared and extremely small. Higgs mediated contributions to these decays do not, in general, suffer from the strong helicity suppression. However, in the SM they are suppressed by the tiny lepton Yukawa couplings and are negligible. One might therefore hope that experiments searching for the $B_{q} \rightarrow e^{+} e^{-}$decays are sensitive to an enhanced Higgs-electron coupling. Here we show that the current and expected sensitivities are not competitive with the direct and indirect bounds discussed so far.

The SM predictions for the time integrated $B_{q} \rightarrow e^{+} e^{-}$branching ratios read [31]

$$
\begin{aligned}
& \operatorname{Br}\left(B_{s} \rightarrow e^{+} e^{-}\right)_{\mathrm{SM}}=(8.54 \pm 0.55) \times 10^{-14}, \\
& \operatorname{Br}\left(B_{d} \rightarrow e^{+} e^{-}\right)_{\mathrm{SM}}=(2.48 \pm 0.21) \times 10^{-15} .
\end{aligned}
$$

These values are many orders of magnitude below the current experimental constraints set by $\mathrm{CDF}[32]$ at $95 \%$ C.L.

$$
\begin{aligned}
& \operatorname{Br}\left(B_{s} \rightarrow e^{+} e^{-}\right)<2.8 \times 10^{-7}, \\
& \operatorname{Br}\left(B_{d} \rightarrow e^{+} e^{-}\right)<8.3 \times 10^{-8} .
\end{aligned}
$$

While the experimental constraints are likely to be improved at LHCb and Belle II by one or two orders of magnitude, sensitivities to the SM predictions will not be reached within the foreseeable future.

In the presence of an enhanced Higgs-electron coupling, we find for the Higgs-mediated correction to the branching ratios ${ }^{5}$

$$
\frac{\operatorname{Br}\left(B_{q} \rightarrow e^{+} e^{-}\right)}{\operatorname{Br}\left(B_{q} \rightarrow e^{+} e^{-}\right)_{\mathrm{SM}}}-1 \propto \frac{m_{B_{q}}^{4}}{M_{h}^{4}} \kappa_{e}^{2},
$$

with a proportionality factor that is parametrically of order 1 . This implies that significant enhancements of the branching ratios are only possible for $\kappa_{e} \gg M_{h}^{2} / m_{B_{q}}^{2} \sim 550$. The current experimental constraints on $B_{q} \rightarrow e^{+} e^{-}$probe couplings of the order of $\kappa_{e} \sim O\left(10^{6}\right)$ that are already excluded by orders of magnitude by the LHC results on $h \rightarrow e^{+} e^{-}$.

\section{Discussion and conclusions}

The question "what do we know about the electron Yukawa" is both interesting and nontrivial to answer.

NP effects could lead to significant changes to the Higgs coupling to electrons precisely because it is predicted to be tiny in the SM. Enhancements of the coupling by orders of magnitude above the SM value are theoretically possible, however only at the cost of significant fine tuning of the electron mass. Order one changes to both the real and imaginary parts of the coupling could be completely natural.

As a side effect, direct verification of an enhanced coupling of the Higgs to electrons would also lead to stronger indirect constraints on $\mathrm{CP}$ violating couplings of the Higgs boson to top quarks [22].

\footnotetext{
${ }^{5}$ Here we assume that $\kappa_{e}$ does not contain a CP violating phase. As discussed in section 4.1, such a phase is strongly constrained by the electron EDM.
} 


\begin{tabular}{|clll|}
\hline \multirow{2}{*}{$h \rightarrow e^{+} e^{-}$} & LHC8 $(25 / \mathrm{fb})$ & $\left|\kappa_{e}\right| \lesssim 600$ & $M \gtrsim 6 \mathrm{TeV}$ \\
& $\operatorname{LHC14}(300 / \mathrm{fb})$ & $\left|\kappa_{e}\right| \sim 260$ & $M \sim 9 \mathrm{TeV}$ \\
& $\operatorname{LHC14}(3 / \mathrm{ab})$ & $\left|\kappa_{e}\right| \sim 150$ & $M \sim 12 \mathrm{TeV}$ \\
& $100 \mathrm{TeV}(3 / \mathrm{ab})$ & $\left|\kappa_{e}\right| \sim 75$ & $M \sim 17 \mathrm{TeV}$ \\
\hline \multirow{2}{*}{$e^{+} e^{-} \rightarrow h$} & LEP II & $\left|\kappa_{e}\right| \lesssim 2000$ & $M \gtrsim 3 \mathrm{TeV}$ \\
& $\operatorname{TLEP}(1 / \mathrm{fb})$ & $\left|\kappa_{e}\right| \sim 50$ & $M \sim 20 \mathrm{TeV}$ \\
& $\operatorname{TLEP}(100 / \mathrm{fb})$ & $\left|\kappa_{e}\right| \sim 10$ & $M \sim 50 \mathrm{TeV}$ \\
\hline \multirow{2}{*}{$d_{e}$} & current & $\operatorname{Im} \kappa_{e} \lesssim 0.017$ & $M \gtrsim 1000 \mathrm{TeV}$ \\
& future & $\operatorname{Im} \kappa_{e} \sim 0.0001$ & $M \sim 10^{4} \mathrm{TeV}$ \\
$(g-2)_{e}$ & current & $\operatorname{Re} \kappa_{e} \lesssim 3000$ & $M \gtrsim 2.5 \mathrm{TeV}$ \\
& future & $\operatorname{Re} \kappa_{e} \sim 300$ & $M \sim 8 \mathrm{TeV}$ \\
\hline
\end{tabular}

Table 2. Summary of current constraints and future expected sensitivities to a modified Higgselectron coupling $\kappa_{e}$ and the corresponding new physics scale $M$.

In this article, we considered which experiments currently provide the most stringent bounds on anomalous Higgs-electron couplings. We find that the strongest bound on the magnitude of the coupling comes from a CMS search for the $h \rightarrow e^{+} e^{-}$decay. The CP-violating imaginary part of the Higgs-electron coupling is strongly constrained by the current upper bound on the electron EDM. The indirect constraint on the CP-conserving real part of the coupling from the electron $g-2$, on the other hand, is currently relatively weak; it can, however, be improved by a new generation of precision experiments and could be competitive with the bounds derived from future LHC data. Finally, we showed that rare $B$ decays are not competitive in setting bounds on deviations from the SM Higgs-electron coupling.

Potentially the best future bounds on the magnitude of the coupling could be obtained from an electron-positron collider running on the Higgs resonance. With optimistic assumptions a measurement of the Higgs-electron coupling only an order of magnitude above its SM value seems possible. Sensitivity to the SM value itself would require huge amounts of statistics collected at the Higgs resonance, very precise knowledge of the Higgs mass of the order of the Higgs width, and exquisite control of the beam energy at the same level. It does not seem that precision measurements of the magnitude of the SM electron Yukawa coupling will ever be possible.

We summarize the current constraints and future expected sensitivities to a modified Higgs-electron coupling $\kappa_{e}$ and the corresponding new physics scale $M$ in table 2 .

\section{Acknowledgments}

This work was initiated at the Aspen Center for Physics with partial support from the National Science Foundation, Grant No. PHYS-1066293. J.B. acknowledges insightful discussions with Felix Yu, and support by the U.S. National Science Foundation under CAREER Grant PHY-1151392, the ERC Advanced Grant EFT4LHC of the European Re- 
search Council, and the Cluster of Excellence Precision Physics, Fundamental Interactions and Structure of Matter (PRISMA-EXC 1098). M.S. would like to thank Andy Cohen for helpful discussions and the US Department of Energy Office of Science for support under Award DE-SC-0010025. Research at Perimeter Institute is supported by the Government of Canada through Industry Canada and by the Province of Ontario through the Ministry of Economic Development \& Innovation.

\section{A Two-loop contributions to dipole moments}

In this appendix we give the analytic expressions for the complete set of relevant two-loop contributions to the electron dipole moments that are induced by a modified Higgs-electron coupling. For the case $\kappa_{e}=1$ we reproduce exactly the part of the bosonic contributions in ref. [33] that involve the exchange of a virtual Higgs boson. To our knowledge, this constitutes the first independent (partial) check of their calculation. For an imaginary value of $\kappa_{e}$ our results for the top-loop diagrams with an internal photon are in agreement with the classic calculation by Barr and Zee [24], while the corresponding analytic results with an internal $Z$ boson are new. Results for the considered bosonic diagrams, in terms of parametric integrals, can in principle be extracted from ref. [34, 35] that give results for two-loop contributions to EDMs in multi-Higgs doublet models (see also [36] for a recent reevaluation of the Barr-Zee type contributions in two-Higgs doublet models). We find small numerical discrepancies with the results of $[34,35]$ of the order of $10 \%$.

To obtain our results we performed an off-shell matching calculation, along the lines of ref. [37], to an effective theory where all heavy particles (the top quark and the $W, Z$, and Higgs bosons) are integrated out. The two physical operators, yielding the magnetic and electric dipole moments in the non-relativistic limit, can be chosen as

$$
\mathcal{O}_{m}=e \bar{\psi}_{e} \sigma^{\mu \nu} \psi_{e} F_{\mu \nu}, \quad \mathcal{O}_{e}=e \bar{\psi}_{e} \sigma^{\mu \nu} i \gamma_{5} \psi_{e} F_{\mu \nu} .
$$

In order to project on the physical matrix elements, we also need the following two operators that vanish via the electron equations of motion:

$$
\mathcal{O}_{m}^{\text {e.o.m. }}=\bar{\psi}_{e} \not D \not D \psi_{e}, \quad \mathcal{O}_{e}^{\text {e.o.m. }}=\bar{\psi}_{e} \not D \not D i \gamma_{5} \psi_{e} .
$$

In our calculation we set the electron mass to zero while keeping the electron Yukawa nonzero. Therefore, no other off-shell operators can contribute at this order, and it is sufficient to expand the integrands to first order in the external momenta.

We have calculated all Feynman diagrams employing the background field gauge for the electroweak interactions [38]. The two-loop integrals were computed using the recursion relations in $[37,39]$. We decompose our result for the two-loop electron EDM in the following way (cf. eq. (4.1))

$$
d_{e}^{2 \text { loop }}=d_{e}^{t \gamma}+d_{e}^{t Z}+d_{e}^{W \gamma}+d_{e}^{W Z}+d_{e}^{W}+d_{e}^{Z} .
$$

The first four terms denote contributions from Barr-Zee type diagrams [24] containing top-quark loops and a photon $\left(d_{e}^{t \gamma}\right)$, top-quark loops and a $Z$ boson $\left(d_{e}^{t Z}\right), W$ boson loops and a photon $\left(d_{e}^{W \gamma}\right)$, and $W$ boson loops and a $Z$ boson $\left(d_{e}^{W Z}\right)$ (see the left and center diagrams in figure 4 for examples). The last two terms in (A.3) denote the remaining 
two-loop contributions that contain either $W$ bosons $\left(d_{e}^{W}\right)$, or $Z$ bosons $\left(d_{e}^{Z}\right)$ (see the right diagram in figure 4 for an example). We obtain for the individual contributions

$$
\begin{aligned}
& \frac{d_{e}^{t \gamma}}{e}=\frac{16 e^{2}}{3\left(16 \pi^{2}\right)^{2}} \frac{y_{e}^{\mathrm{SM}}}{\sqrt{2} v} \operatorname{Im} \kappa_{e} x_{t h}\left[\left(2 x_{t h}-1\right) \Phi\left(\frac{1}{4 x_{t h}}\right)-2\left(2+\log x_{t h}\right)\right], \\
& \frac{d_{e}^{t Z}}{e}=\frac{e^{2}}{\left(16 \pi^{2}\right)^{2} s_{w}^{2}} \frac{y_{e}^{\mathrm{SM}}}{\sqrt{2} v} \operatorname{Im} \kappa_{e} \frac{1}{2 c_{w}^{2}}\left(1-4 s_{w}^{2}\right)\left(1-\frac{8}{3} s_{w}^{2}\right)\left(1-x_{h Z}\right)^{-1} x_{t Z} \\
& \times\left[\left(1-2 x_{t h}\right) \Phi\left(\frac{1}{4 x_{t h}}\right)-2 \log x_{h Z}-\left(1-2 x_{t Z}\right) \Phi\left(\frac{1}{4 x_{t Z}}\right)\right], \\
& \frac{d_{e}^{W \gamma}}{e}=\frac{2 e^{2}}{\left(16 \pi^{2}\right)^{2}} \frac{y_{e}^{\mathrm{SM}}}{\sqrt{2} v} \operatorname{Im} \kappa_{e}\left[\left(1+6 x_{W h}\right)\left(2+\log x_{W h}\right)\right. \\
& \left.-\left(6 x_{W h}-7\right) x_{W h} \Phi\left(\frac{1}{4 x_{W h}}\right)\right], \\
& \frac{d_{e}^{W Z}}{e}=\frac{e^{2}}{\left(16 \pi^{2}\right)^{2} s_{w}^{2}} \frac{y_{e}^{\mathrm{SM}}}{4 \sqrt{2} v} \operatorname{Im} \kappa_{e}\left(1-4 s_{w}^{2}\right)\left(1-x_{Z h}\right)^{-1} \\
& \times\left[\left(2+12 x_{W h}-\frac{1}{c_{w}^{2}}-2 x_{Z h}\right) \log x_{Z h}\right. \\
& +\left(14-12 x_{W h}-\frac{3}{c_{w}^{2}}+2 x_{Z h}\right) x_{W h} \Phi\left(\frac{1}{4 x_{W h}}\right) \\
& \left.+\left(2+12 x_{W h}-\frac{1}{c_{w}^{2}}+\frac{4 x_{Z h}}{c_{w}^{2}}-18 x_{Z h}\right) c_{w}^{2} \Phi\left(\frac{1}{4 c_{w}^{2}}\right)\right] . \\
& \frac{d_{e}^{W}}{e}=\frac{e^{2}}{\left(16 \pi^{2}\right)^{2}} \frac{y_{e}^{\mathrm{SM}}}{18 \sqrt{2} v} \frac{1}{s_{w}^{2}} \operatorname{Im} \kappa_{e} x_{h W} \\
& \times\left\{6\left(x_{W h}^{2}+4 x_{W h}-2\right) \Phi\left(\frac{1}{4 x_{W h}}\right)-6\left(4 x_{W h}^{3}+3 x_{W h}^{2}-4\right) \operatorname{Li}_{2}\left(1-x_{W h}\right)\right. \\
& -\pi^{2} x_{W h}^{2}\left(3+4 x_{W h}\right)+24 x_{W h}\left(x_{W h}-1\right)+24 x_{W h}\left(x_{W h}+1\right) \log x_{W h} \\
& \left.-3\left(4 x_{W h}^{3}+3 x_{W h}^{2}-4\right) \log ^{2} x_{W h}\right\} \text {, } \\
& \frac{d_{e}^{Z}}{e}=\frac{e^{2}}{\left(16 \pi^{2}\right)^{2}} \frac{y_{e}^{\mathrm{SM}}}{36 \sqrt{2} v} \frac{1}{s_{w}^{2} c_{w}^{2}} \operatorname{Im} \kappa_{e} x_{h Z}\left(8 c_{w}^{4}-12 c_{w}^{2}+5\right) \\
& \times\left\{-6\left(4 x_{Z h}^{3}+3 x_{Z h}^{2}-1\right) \operatorname{Li}_{2}\left(1-x_{Z h}\right)-3\left(1-2 x_{Z h}-8 x_{Z h}^{2}\right) \Phi\left(\frac{1}{4 x_{Z h}}\right)\right. \\
& -\pi^{2} x_{Z h}^{2}\left(3+4 x_{Z h}\right)-6 x_{Z h}\left(1-4 x_{Z h}\right)+6 x_{Z h}\left(1+4 x_{Z h}\right) \log x_{Z h} \\
& \left.-3\left(4 x_{Z h}^{3}+3 x_{Z h}^{2}-1\right) \log ^{2} x_{Z h}\right\} \\
& +\frac{e^{2}}{\left(16 \pi^{2}\right)^{2}} \frac{y_{e}^{\mathrm{SM}}}{6 \sqrt{2} v} \frac{1}{c_{w}^{2}} \operatorname{Im} \kappa_{e}\left(s_{w}^{2}-c_{w}^{2}\right) x_{h Z}^{3} \\
& \times\left\{12\left(1-4 x_{Z h}+x_{Z h}^{2}\right) \operatorname{Li}_{2}\left(1-x_{Z h}\right)-3\left(1-6 x_{Z h}+8 x_{Z h}^{2}\right) \Phi\left(\frac{1}{4 x_{Z h}}\right)\right. \\
& -\pi^{2}\left(1-4 x_{Z h}\right)-6 x_{Z h}^{2}+12 x_{Z h}^{2} \log x_{Z h} \\
& \left.+3\left(2 x_{Z h}^{2}-4 x_{Z h}+1\right) \log ^{2} x_{Z h}\right\} \text {. }
\end{aligned}
$$


To simplify the expressions we defined the mass ratios $x_{i j} \equiv M_{i}^{2} / M_{j}^{2}, c_{w}=M_{W} / M_{Z}$, and $s_{w}=\sqrt{1-c_{w}^{2}}$. The function $\Phi(z)$ is given by [39]

$$
\begin{aligned}
\Phi(z) & =4\left(\frac{z}{1-z}\right)^{1 / 2} \mathrm{Cl}_{2}\left(2 \arcsin \left(z^{1 / 2}\right)\right) \\
\mathrm{Cl}_{2}(\theta) & =-\int_{0}^{\theta} d x \log |2 \sin (x / 2)|
\end{aligned}
$$

for $z<1$ and by

$$
\begin{aligned}
\Phi(z) & =\left(\frac{z}{z-1}\right)^{1 / 2}\left\{-4 \operatorname{Li}_{2}(\xi)+2 \log ^{2} \xi-\log ^{2}(4 z)+\frac{\pi^{2}}{3}\right\}, \\
\xi & =\frac{1-\left(\frac{z-1}{z}\right)^{1 / 2}}{2},
\end{aligned}
$$

for $z>1$, where $\operatorname{Li}_{2}(x)=-\int_{0}^{x} d u \ln (1-u) / u$ is the usual dilogarithm. The numerical size of the individual contributions is

$$
\begin{aligned}
\frac{d_{e}^{2 \text { loop }}}{e}=\frac{d_{e}^{t \gamma}}{e}+\frac{d_{e}^{t Z}}{e}+\frac{d_{e}^{W \gamma}}{e} & +\left(\frac{d_{e}^{W Z}}{e}+\frac{d_{e}^{W}}{e}+\frac{d_{e}^{Z}}{e}\right) \\
& =\operatorname{Im} \kappa_{e} \times(-6.44-0.12+13.85-2.22) \times 10^{-27} \mathrm{~cm}
\end{aligned}
$$

Note that the Barr-Zee contributions involving $Z$ bosons are suppressed by the small vector coupling of the $Z$ boson to leptons proportional to $\left(1-4 s_{w}^{2}\right)$.

We checked explicitly that the corresponding contributions to the anomalous magnetic moment of the electron can be obtained via

$$
\Delta a_{e}=\frac{\left(\operatorname{Re} \kappa_{e}-1\right)}{\operatorname{Im} \kappa_{e}} 2 m_{e}\left(\frac{d_{e}}{e}\right) .
$$

The results of this appendix can also easily be adapted to obtain expressions for flavor violating dipole transitions such as $\mu \rightarrow e \gamma$ in the presence of flavor-violating Higgs couplings.

\section{B Enhanced Higgs production through a loop hole?}

Naively one might expect that, in the SM, higher-order Feynman graphs (for example figure 5) could lead to enhanced $s$-channel Higgs production, not suppressed by the small Yukawa coupling. Here we show that this expectation is wrong. To see this note that in the limit of vanishing electron Yukawa coupling (and ignoring neutrino masses), the SM Lagrangian has an exact enhanced (chiral) symmetry rotating the left- and right-handed components of the electron field. Thus any non-vanishing amplitude of electrons coupling to the Higgs must either be proportional to the electron Yukawa coupling (as we want to show) or preserve chiral symmetry. But any chiral symmetry preserving coupling of electrons has the electrons combined into a vector which must be dotted into a electron momentum to form a Lorentz invariant amplitude. Then the electron equation of motion can be used to turn the momentum into the electron mass. 

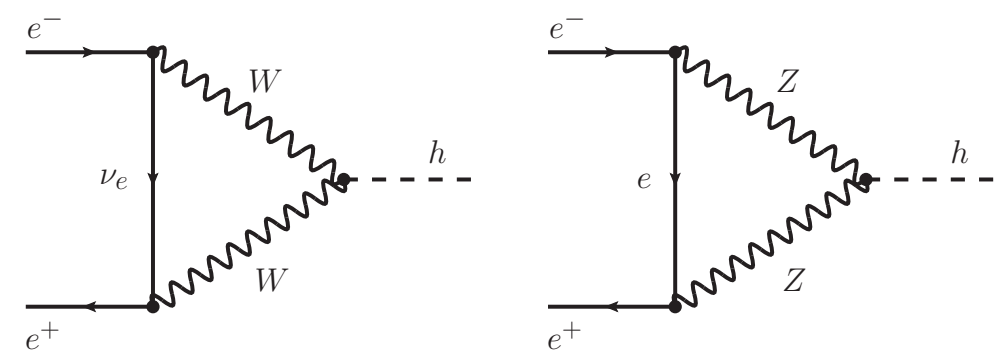

Figure 5. Sample one-loop Feynman diagrams which naively look like they might give a SM $s$ channel Higgs production cross section that is not suppressed by the small electron Yukawa. As shown in the text, chiral symmetry implies that the amplitude for this process is suppressed by the electron mass for on-shell external fermions.

To make this argument more explicit, consider the amplitude for the transition of an onshell electron-positron pair into a (not necessarily on-shell) Higgs boson (the argument for the reverse process is very similar). Its most general Lorentz spinor structure is of the form

$$
\bar{v}_{e}\left(\mathbf{p}^{\prime}, \sigma^{\prime}\right)\left[A+B \gamma_{5}+C_{\mu} \gamma^{\mu}+D_{\mu} \gamma^{\mu} \gamma_{5}+E_{\mu \nu} \sigma^{\mu \nu}\right] u_{e}(\mathbf{p}, \sigma)
$$

where $A, B, C_{\mu}, D_{\mu}, E_{\mu \nu}$ are coefficients which must be constructed out of the Lorentz invariants $p^{2}=p^{\prime 2}=m_{e}^{2}, p \cdot p^{\prime}$ and the two independent Lorentz vectors $p_{\mu}$ and $p_{\mu}^{\prime}$.

The amplitudes proportional to $A, B, E_{\mu \nu}$ violate chiral symmetry, thus they can only be generated proportional to the electron Yukawa coupling. The amplitudes with $C_{\mu}$ and $D_{\mu}$ preserve chiral symmetry and need not be suppressed. However, by Lorentz symmetry $C_{\mu}$ and $D_{\mu}$ must be proportional to either $p_{\mu}$ or $p_{\mu}^{\prime}$. Thus we obtain amplitudes of the form $\bar{v}_{e}\left(\mathbf{p}^{\prime}\right) \not p u_{e}(\mathbf{p})$ and $\bar{v}_{e}\left(\mathbf{p}^{\prime}\right) \not p^{\prime \prime} u_{e}(\mathbf{p})$ which are proportional to $m_{e}$ by the equations of motion for on-shell external electrons. Therefore, $s$-channel Higgs production in electron-positron collisions is always suppressed by at least one power of $m_{e}$ (and possible loop factors).

This general argument based on spin and Lorentz invariance continues to apply for amplitudes with additional soft photons which cannot carry angular momentum. However amplitudes with additional hard photons need not be suppressed by the electron mass. For example, the process $e^{+} e^{-} \rightarrow \gamma^{*} \rightarrow \gamma h$ does arise in the SM and is not suppressed by the electron mass. However it is suppressed by the small loop-induced coupling of the Higgs to photons and was therefore not relevant for Higgs production at LEP2. The presence of the hard photon in the final state would of course allow experimenters to distinguish this process from the s-channel Higgs production process in attempts to measure the Higgselectron coupling. 
Open Access. This article is distributed under the terms of the Creative Commons Attribution License (CC-BY 4.0), which permits any use, distribution and reproduction in any medium, provided the original author(s) and source are credited.

\section{References}

[1] CMS collaboration, Precise determination of the mass of the Higgs boson and tests of compatibility of its couplings with the standard model predictions using proton collisions at 7 and $8 \mathrm{TeV}$, arXiv: 1412.8662 [INSPIRE].

[2] ATLAS collaboration, Updated coupling measurements of the Higgs boson with the ATLAS detector using up to $25 \mathrm{fb}^{-1}$ of proton-proton collision data, ATLAS-CONF-2014-009 (2014).

[3] G.T. Bodwin, F. Petriello, S. Stoynev and M. Velasco, Higgs boson decays to quarkonia and the Hc̄c coupling, Phys. Rev. D 88 (2013) 053003 [arXiv:1306.5770] [InSPIRE].

[4] C. Delaunay, T. Golling, G. Perez and Y. Soreq, Enhanced Higgs boson coupling to charm pairs, Phys. Rev. D 89 (2014) 033014 [arXiv:1310.7029] [INSPIRE].

[5] A.L. Kagan et al., Exclusive Window onto Higgs Yukawa Couplings, Phys. Rev. Lett. 114 (2015) 101802 [arXiv:1406.1722] [INSPIRE].

[6] G.F. Giudice and O. Lebedev, Higgs-dependent Yukawa couplings, Phys. Lett. B 665 (2008) 79 [arXiv: 0804.1753] [INSPIRE].

[7] CMS collaboration, Search for a standard model-like Higgs boson in the $\mu^{+} \mu^{-}$and $e^{+} e^{-}$ decay channels at the LHC, Phys. Lett. B 744 (2015) 184 [arXiv:1410.6679] [INSPIRE].

[8] Particle Data Group collaboration, K. Olive et al., Review of Particle Physics, Chin. Phys. C 38 (2014) 090001.

[9] LHC Higgs Cross section Working Group collaboration, S. Heinemeyer et al., Handbook of LHC Higgs Cross sections: 3. Higgs Properties, arXiv:1307.1347 [INSPIRE].

[10] LHC Higgs Cross section Working Group collaboration, S. Dittmaier et al., Handbook of LHC Higgs Cross sections: 1. Inclusive Observables, arXiv:1101.0593 [INSPIRE].

[11] https://twiki.cern.ch/twiki/bin/view/LHCPhysics/HiggsEuropeanStrategy2012\# SM_Higgs_production_cross_se_AN2.

[12] ALEPH, DELPHI, L3, OPAL collaborations, LEP Electroweak Working Group, J. Alcaraz et al., A combination of preliminary electroweak measurements and constraints on the standard model, hep-ex/0612034 [INSPIRE].

[13] J. Alwall et al., The automated computation of tree-level and next-to-leading order differential cross sections and their matching to parton shower simulations, JHEP 07 (2014) 079 [arXiv: 1405.0301] [INSPIRE].

[14] J.J. Ward, Precision measurements of particle masses using jets at LEP-2, hep-ex/9912025 [INSPIRE].

[15] T. Han and Z. Liu, Potential precision of a direct measurement of the Higgs boson total width at a muon collider, Phys. Rev. D 87 (2013) 033007 [arXiv:1210.7803] [INSPIRE].

[16] TLEP Design Study Working Group collaboration, M. Bicer et al., First Look at the Physics Case of TLEP, JHEP 01 (2014) 164 [arXiv:1308.6176] [INSPIRE]. 
[17] M. Consoli, W. Hollik and F. Jegerlehner, Electroweak radiative corrections for $Z$ physics, CERN-TH-5527-89 [INSPIRE].

[18] G. Blankenburg, J. Ellis and G. Isidori, Flavour-Changing Decays of a $125 \mathrm{GeV}$ Higgs-like Particle, Phys. Lett. B $\mathbf{7 1 2}$ (2012) 386 [arXiv:1202.5704] [INSPIRE].

[19] R. Harnik, J. Kopp and J. Zupan, Flavor Violating Higgs Decays, JHEP 03 (2013) 026 [arXiv: 1209.1397] [INSPIRE].

[20] M. Gorbahn and U. Haisch, Searching for $t \rightarrow c(u) h$ with dipole moments, JHEP 06 (2014) 033 [arXiv: 1404.4873] [INSPIRE].

[21] D. McKeen, M. Pospelov and A. Ritz, Modified Higgs branching ratios versus CP and lepton flavor violation, Phys. Rev. D 86 (2012) 113004 [arXiv:1208.4597] [INSPIRE].

[22] J. Brod, U. Haisch and J. Zupan, Constraints on CP-violating Higgs couplings to the third generation, JHEP 11 (2013) 180 [arXiv:1310.1385] [INSPIRE].

[23] W. Altmannshofer, M. Bauer and M. Carena, Exotic Leptons: Higgs, Flavor and Collider Phenomenology, JHEP 01 (2014) 060 [arXiv: 1308.1987] [INSPIRE].

[24] S.M. Barr and A. Zee, Electric Dipole Moment of the Electron and of the Neutron, Phys. Rev. Lett. 65 (1990) 21 [inSPIRE].

[25] ACME collaboration, J. Baron et al., Order of Magnitude Smaller Limit on the Electric Dipole Moment of the Electron, Science 343 (2014) 269 [arXiv:1310.7534] [INSPIRE].

[26] J.L. Hewett et al., Fundamental Physics at the Intensity Frontier, arXiv:1205.2671 [INSPIRE].

[27] D. Hanneke, S. Fogwell and G. Gabrielse, New Measurement of the Electron Magnetic Moment and the Fine Structure Constant, Phys. Rev. Lett. 100 (2008) 120801 [arXiv: 0801.1134] [INSPIRE].

[28] T. Aoyama, M. Hayakawa, T. Kinoshita and M. Nio, Tenth-Order Electron Anomalous Magnetic Moment - Contribution of Diagrams without Closed Lepton Loops, Phys. Rev. D 91 (2015) 033006 [arXiv:1412.8284] [INSPIRE].

[29] G.F. Giudice, P. Paradisi and M. Passera, Testing new physics with the electron g-2, JHEP 11 (2012) 113 [arXiv:1208.6583] [inSPIRE].

[30] R. Bouchendira, P. Clade, S. Guellati-Khelifa, F. Nez and F. Biraben, New determination of the fine structure constant and test of the quantum electrodynamics, Phys. Rev. Lett. 106 (2011) 080801 [arXiv: 1012.3627] [INSPIRE].

[31] C. Bobeth et al., $B_{s, d} \rightarrow \ell^{+} \ell^{-}$in the Standard Model with Reduced Theoretical Uncertainty, Phys. Rev. Lett. 112 (2014) 101801 [arXiv:1311.0903] [INSPIRE].

[32] CDF collaboration, T. Aaltonen et al., Search for the Decays $B_{s}^{0} \rightarrow e^{+} \mu^{-}$and $B_{s}^{0} \rightarrow e^{+} e^{-}$ in CDF Run II, Phys. Rev. Lett. 102 (2009) 201801 [arXiv:0901.3803] [INSPIRE].

[33] T. Gribouk and A. Czarnecki, Electroweak interactions and the muon g-2: Bosonic two-loop effects, Phys. Rev. D 72 (2005) 053016 [hep-ph/0509205] [INSPIRE].

[34] R.G. Leigh, S. Paban and R.M. Xu, Electric dipole moment of electron, Nucl. Phys. B 352 (1991) 45 [INSPIRE].

[35] D. Chang, W.-Y. Keung and T.C. Yuan, Two loop bosonic contribution to the electron electric dipole moment, Phys. Rev. D 43 (1991) 14 [INSPIRE]. 
[36] T. Abe, J. Hisano, T. Kitahara and K. Tobioka, Gauge invariant Barr-Zee type contributions to fermionic EDMs in the two-Higgs doublet models, JHEP 01 (2014) 106 [arXiv:1311.4704] [INSPIRE].

[37] C. Bobeth, M. Misiak and J. Urban, Photonic penguins at two loops and $m(t)$ dependence of $B R\left[B \rightarrow X(s) \ell^{+} \ell^{-}\right]$, Nucl. Phys. B 574 (2000) 291 [hep-ph/9910220] [InSPIRE].

[38] A. Denner, G. Weiglein and S. Dittmaier, Application of the background field method to the electroweak standard model, Nucl. Phys. B 440 (1995) 95 [hep-ph/9410338] [INSPIRE].

[39] A.I. Davydychev and J.B. Tausk, Two loop selfenergy diagrams with different masses and the momentum expansion, Nucl. Phys. B 397 (1993) 123 [INSPIRE]. 